\title{
Early Detection is as Important as Imatinib in CML Treatment Success
}

Authors: Dmitriy Sonkin and Richard Simon

National Cancer Institute, Division of Cancer Treatment and Diagnosis, Biometric Research Program, Rockville, MD, USA

Correspondence should be addressed to: dmitriy.sonkin@nih.gov, rsimon@mail.nih.gov

Competing financial interests statement: None

\begin{abstract}
Chronic myelogenous leukemia (CML) was the first malignancy for which clinical outcome was drastically improved by kinase inhibitor therapy. Kinase inhibitors targeting other well-known oncogenes have been introduced into clinical practice, but none have shown the same magnitude of clinical benefit as ABL1 inhibition in CML. We argue that early detection is an underappreciated, but critically important factor in success of ABL1 inhibitors in treatment of CML. We show that CML provides a window into how many types of cancer may look and behave at an early stage, prior to diagnosis and the development of additional genomic alterations. The remarkable clinical benefits of ABL1 inhibition is likely due to early detection of $\mathrm{CML}$ at a stage in which the tumor is driven by single oncogenic alteration which can be successfully controlled by the inhibitor. Thinking of CML as a prototype for effective systemic treatment based on early cancer detection may help to develop strategies for improving treatment for other types of cancer.
\end{abstract}

Keywords: CML; Imatinib; early detection; cancer; tumor progression; oncology; Gleevec; Glivec 


\section{Introduction}

Chronic myelogenous leukemia (CML) research, conducted over a period of more than 50 years, has provided important insights into CML biology ${ }^{1,2,3}$ and cancer biology in general ${ }^{4}$. The identification and functional characterization of the BCR-ABL fusion eventually led to development of the ABL1 tyrosine kinase inhibitor STI571 (Signal Transduction Inhibitor number 571) which is currently known as Gleevec or Imatinib ${ }^{5}$. Introduction of Imatinib into clinical practice, about 20 years ago, markedly improved the prognosis for chronic phase CML patients. The IRIS study reported an $86 \%$ overall survival for patients on Imatinib after 7 years on therapy (93\% if only CML related deaths are considered) ${ }^{6}$. The ILTE study of patients on Imatinib who were in CCyR (complete cytogenetic response) after 2 years, reported only $1 \%$ of CML related deaths 8 years after start of Imatinib treatment ${ }^{7}$.

Prior to the introduction of Imatinib, the majority of CML patients would progress to blast crisis ${ }^{8,9}$ and the median overall survival of CML patients was around 6 years ${ }^{10}$. In the Imatinib era, the vast majority of CML patients do not progress to blast crisis and have excellent long term survival (approaching age matched population) ${ }^{7,11}$. Unfortunately, a fraction of CML patients still progress to blast crisis and even today the median survival after the onset of blast crisis is just around 9 months, not much better than in pre Imatinib era ${ }^{12}$.

\section{Early detection and treatment}

Clearly, as indicated above, $C M L$ is highly deadly disease and yet $A B L 1$ inhibitors are able to put CML into deep and essentially fully controlled remission in majority of patients and even potentially completely eradicate disease in $\sim 10 \%-15 \%$ of patients ${ }^{13,14}$. So why are ABL1 inhibitors so remarkably successful in CML treatment?

A key factor in the successful management of CML is early detection. Here "early" means at a time when the disease is driven by a single druggable genomic alteration, before the development of other genomic alterations which drive tumor progression. In developed countries $\mathrm{CML}$ is diagnosed in chronic phase in $90 \%$ of patients; half of them are asymptomatic and diagnosis is due to blood tests performed for routine physical or other $\mathrm{CML}$ unrelated reasons ${ }^{9}$.

Patients promptly diagnosed with chronic phase CML have a malignancy which, in the majority of cases, is driven by single alteration, BCR-ABL fusion. In most of such cases overactive ABL1 kinase activity can be controlled by ABL1 kinase inhibitors. Successful inhibition of ABL1 kinase activity leads to elimination of the majority of leukemic cells with BCR-ABL fusion ${ }^{15,16}$. The ENESTnd trial ${ }^{17}$ showed that at 5 years $77 \%$ of patients treated with the second generation ABL1 inhibitor nilotinib reached major molecular response (MMR) which corresponds to 1000 times decrease in BCR-ABL fusion levels. As a result of such a profound decrease in the number of cells with the BCR-ABL fusion there is drastic reduction in number of cells which available to potentially accumulate additional alterations leading to a greatly decreased risk of progression to blast crisis. 


\section{CML progression to blast crisis}

Progression to blast crisis CML (CML-BC) is associated with accumulation of additional genomic alterations $^{4,9}$. New genetic alterations have been detected in around $80 \%$ of CML-BC patients ${ }^{9,18}$ and the actual percentage is probably higher due to the fact that most of the studies were not able to screen for alteration in all relevant genes. About 30\% of CML-BC patients have TP53 inactivating alterations ${ }^{9}$, RUNX1 alterations are detected in around $35 \%{ }^{18}, \mathrm{CDKN} 2 \mathrm{~A} / \mathrm{B}$ inactivating alterations are found in around $13 \%$ and IKZF1 inactivating alterations are found in around $15 \%{ }^{9}$. CDKN2A/B and IKZF1 inactivating alterations are mostly limited to lymphoid blast crisis which occurs in about $25 \%$ of CML-BC patients ${ }^{9}$. There are also alterations found in other cancer relevant genes, but at lower frequency ${ }^{18,4}$.

TP53 and CDKN2A/B are very well known tumor suppressor genes altered in a wide variety of solid tumors and hematopoietic malignancies 19,20,21. IKZF1 and RUNX1 are well known for their role in hematopoiesis and hematopoietic malignancies ${ }^{22,23,24,25}$. Alterations in TP53 ${ }^{26}$, CDKN2A/B ${ }^{27}$, IKZF1 ${ }^{28}$, and RUNX1 ${ }^{23}$ are rarely detected in CML chronic phase ${ }^{29}$. For example, in the study of $42 \mathrm{CML}$ patients for which paired DNA samples were available for chronic phase and blast crisis, CDKN2A deletions were detected in $29 \%$ of samples with lymphoid CML-BC and not observed at all in chronic phase ${ }^{30}$. TP53, CDKN2A/B, IKZF1 and RUNX1 are tumor suppressors and/or transcription factors and currently there is essentially no successful therapy to substitute for loss of function of these genes. It is not completely clear mechanistically why mutations in these genes reduce the sensitivity of CML cells to ABL1 kinase inhibitors. One possibility is that the apoptosis response to damage or cellular stress is lost or diminished. A second possibility is that loss of DNA repair or cell cycle checkpoints permits the accumulation of mutations which drive proliferation regardless of $A B L 1$ inhibition. $A$ third possibility is that the accumulation of mutations results in a re-wiring of the expression program of CML cells making ABL1 status irrelevant or less critical. A re-wiring of the expression program is supported by the fact that alterations in IKZF1 and RUNX1 may impact differentiation and that loss of CDKN2A may contribute to transformation from myeloid to lymphoid malignancy.

\section{Proto-typical malignancy}

Presence of multiple genomic alternations in CML-BC is similar to other hematopoietic and solid tissue malignancies, which at diagnosis are characterized by combinations of alterations in tumor suppressors and oncogenes. The pattern of molecular and clinical progression of CML suggests that CML may not be an atypical malignancy, but instead a window into how many types of cancer perhaps look and behave at an early stage of the disease prior to diagnosis. Adenoma to carcinoma progression of colorectal cancer is one of the most well-studied examples of solid tumor progression and has clear similarities to CML progression. The progression to colorectal carcinoma is estimated to take 10 to 20 years and the dynamics of the progression from the stage of the earliest founder mutation is not generally observable as in the case of CML. Activation of the WNT pathway due to loss of function of APC or gain of function of CTNNB1 may lead to formation of an aberrant crypt. Subsequent mutations in KRAS, NRAS or BRAF promote progression to adenoma, loss of $18 \mathrm{q}$ is associated with advanced adenoma and alterations in genes such as TP53, PIK3CA promote progression to carcinoma. 
Most tumors have experienced many generations of cell division by the time of clinical detection. They often contain 3-5 so called "driver mutations" and may contain numerous other genomic alterations of uncertain functional significance. It is rarely known how many "founder mutations" a tumor contains where a founder mutation indicates that it is one of the early mutations of the cell of origin which makes that cell malignant. Single cell sequencing technologies are making it more feasible to better understand these early stages of oncogenesis. Some of the founder alterations may be inherited, for example germline inactivating variants in RB1, SMARCB1, CDKN2A, PTEN, TP53. Several genetically engineered mouse models (GEMMs) based on just one or two driver/founder alterations have been constructed. For example, there are GEMMs based on alterations in SMARCB1, PTEN and BRAF, RB1 and APC. As noted by Kaelin ${ }^{31}$, the founder alterations appear in all sub-clones of the tumor and so they may be good molecular targets for treatment strategies. Also, since all of the founder mutations were necessary for making the cell of origin malignant, reversal of the effect of only a single founder alteration may be sufficient to reverse or control the malignant phenotype.

As in the case of CML, however after molecular progression inhibition of a single founder mutation may no longer be effective for controlling the disease. For example, during molecular progression of the tumor it is possible that a new sub-clone was formed which is no longer completely dependent on any single founder mutation. The observations above indicate that early detection may be necessary for effective treatment, even for effective systemic treatment. It is important to note that the type of gene effected by first driver mutation will likely have implication on degree of effectiveness of targeted systemic treatment based on early detection. If first driver mutation is in oncogene for which effective targeted treatment is available, like BCR-ABL fusion, it is possible to anticipate good efficacy of treatment. However, if first driver mutation is in tumor suppressor gene or oncogene for which effective targeted treatment is not available, targeted systemic treatment based on early detection would be likely less effective, since at least one un-targetable driver mutation will be present.

CML is not the only example of a malignancy with successful early detection and treatment. Generally, the treatment associated with early detection is surgery or radiotherapy. For example, colorectal cancer incidence and mortality rates in US decreased around 50\% from mid-1980s in part due to successful screening and treatment programs. In the US 5-year survival for ovarian cancer diagnosed before it spreads outside of ovaries is $92 \%$. If there is a spread to surrounding tissue/organs within the pelvis, 5 year survival decreases to $73 \%$. In case of cancer spread beyond pelvis and/or into retroperitoneal lymph nodes 5 -year survival drops to $28 \%$. Unlike CML, which is in most cases detected early, only $15 \%$ of patients are diagnosed prior to cancer spread outside of ovaries and around $75 \%$ of patients present with ovarian cancer which is already spread beyond pelvis.

Recent studies provide clear evidence that, as expected, oncogenic somatic mutations can be found in clonal expansions in overall normal tissues and blood and chance of detecting such mutations increases with age ${ }^{32,33}$. The tissue and cell type of origin of such mutations are likely to be important factors in possibility of such early neoplasms progressing to malignancy. For example, some tissue types may be more sensitive to single oncogenic somatic mutation and others may be more resilient and require more than one oncogenic somatic mutation to have a high risk of progression, it also possible that if a cell 
which acquired oncogenic somatic mutation is a progenitor, the chance of progression to malignancy would be higher.

\section{Conclusion}

The Imatinib profound success in CML treatment is, in part, due to early detection. Effective early cancer detection and treatment is a complicated challenge ${ }^{34,35}$. It involves using technologies and follow up clinical management protocols that provide a sufficiently long diagnostic lead-time while balancing the threat from the underlying malignancy and treatment associated morbidity and low, but not insignificant risk of treatment induced mortality. Recognition of the potential of systemic treatment, rather than just surgery or radiotherapy, at the earliest stages of the disease may in some cases open new opportunities for early detection and treatment strategies. New technologies are continuously improving, ability to detect and analyze circulating cell free tumor DNA in blood, urine and other body fluids may enable detection at much earlier stage of disease than previously possible ${ }^{36}$. Recognition that systemic therapy with agents molecularly targeted to one or perhaps two of the founder mutations may be curative can enhance the effectiveness of early detection even in cases where local eradication is problematic.

\section{References}

1. Nowell PC, Hungerford DA. A minute chromosome in human chronic granulocytic leukemia. Science. 1960;132(3438):1497.

2. Rowley JD. Letter: A new consistent chromosomal abnormality in chronic myelogenous leukaemia identified by quinacrine fluorescence and Giemsa staining. Nature. 1973;243(5405):290-293.

3. McLaughlin J, Chianese $E$, Witte ON. In vitro transformation of immature hematopoietic cells by the P210 BCR/ABL oncogene product of the Philadelphia chromosome. Proc Natl Acad Sci U S A. 1987;84(18):6558-6562.

4. Melo JV, Barnes DJ. Chronic myeloid leukaemia as a model of disease evolution in human cancer. Nat Rev Cancer. 2007;7(6):441-453. doi:10.1038/nrc2147

5. Druker BJ, Talpaz M, Resta DJ, et al. Efficacy and safety of a specific inhibitor of the BCR-ABL tyrosine kinase in chronic myeloid leukemia. N Engl J Med. 2001;344(14):1031-1037. doi:10.1056/NEJM200104053441401

6. Hughes TP, Hochhaus A, Branford S, et al. Long-term prognostic significance of early molecular response to imatinib in newly diagnosed chronic myeloid leukemia: an analysis from the International Randomized Study of Interferon and STI571 (IRIS). Blood. 2010;116(19):3758-3765. doi:10.1182/blood-2010-03-273979

7. Gambacorti-Passerini C, Antolini L, Mahon F-X, et al. Multicenter independent assessment of outcomes in chronic myeloid leukemia patients treated with imatinib. J Natl Cancer Inst. 2011;103(7):553-561. doi:10.1093/jnci/djr060 
8. Kantarjian $\mathrm{H}, \mathrm{O}^{\prime}$ Brien $\mathrm{S}$, Jabbour $\mathrm{E}$, et al. Improved survival in chronic myeloid leukemia since the introduction of imatinib therapy: a single-institution historical experience. Blood.

2012;119(9):1981-1987. doi:10.1182/blood-2011-08-358135

9. Perrotti D, Jamieson C, Goldman J, Skorski T. Chronic myeloid leukemia: mechanisms of blastic transformation. J Clin Invest. 2010;120(7):2254-2264. doi:10.1172/JCI41246

10. Hehlmann R, Heimpel H, Hasford J, et al. Randomized comparison of interferon-alpha with busulfan and hydroxyurea in chronic myelogenous leukemia. The German CML Study Group. Blood. 1994;84(12):4064-4077.

11. Huang X, Cortes J, Kantarjian H. Estimations of the increasing prevalence and plateau prevalence of chronic myeloid leukemia in the era of tyrosine kinase inhibitor therapy. Cancer.

2012;118(12):3123-3127. doi:10.1002/cncr.26679

12. Hehlmann R. How I treat CML blast crisis. Blood. 2012;120(4):737-747. doi:10.1182/blood-201203-380147

13. Mahon F-X, Réa D, Guilhot J, et al. Discontinuation of imatinib in patients with chronic myeloid leukaemia who have maintained complete molecular remission for at least 2 years: the prospective, multicentre Stop Imatinib (STIM) trial. Lancet Oncol. 2010;11(11):1029-1035. doi:10.1016/S1470-2045(10)70233-3

14. Etienne G, Guilhot J, Rea D, et al. Long-Term Follow-Up of the French Stop Imatinib (STIM1) Study in Patients With Chronic Myeloid Leukemia. J Clin Oncol Off J Am Soc Clin Oncol. 2017;35(3):298305. doi:10.1200/JCO.2016.68.2914

15. Soverini S, Hochhaus A, Nicolini FE, et al. BCR-ABL kinase domain mutation analysis in chronic myeloid leukemia patients treated with tyrosine kinase inhibitors: recommendations from an expert panel on behalf of European LeukemiaNet. Blood. 2011;118(5):1208-1215. doi:10.1182/blood-2010-12-326405

16. Soverini S, De Benedittis C, Mancini M, Martinelli G. Best Practices in Chronic Myeloid Leukemia Monitoring and Management. The Oncologist. 2016;21(5):626-633.

doi:10.1634/theoncologist.2015-0337

17. Hochhaus A, Saglio G, Hughes TP, et al. Long-term benefits and risks of frontline nilotinib vs imatinib for chronic myeloid leukemia in chronic phase: 5-year update of the randomized ENESTnd trial. Leukemia. 2016;30(5):1044-1054. doi:10.1038/leu.2016.5

18. Grossmann V, Kohlmann A, Zenger $\mathrm{M}$, et al. A deep-sequencing study of chronic myeloid leukemia patients in blast crisis (BC-CML) detects mutations in 76.9\% of cases. Leukemia. 2011;25(3):557560. doi:10.1038/leu.2010.298

19. Lanza F, Bi S. Role of p53 in leukemogenesis of chronic myeloid leukemia. Stem Cells Dayt Ohio. 1995;13(4):445-452. doi:10.1002/stem.5530130416 
20. Mullighan CG, Williams RT, Downing JR, Sherr CJ. Failure of CDKN2A/B (INK4A/B-ARF)-mediated tumor suppression and resistance to targeted therapy in acute lymphoblastic leukemia induced by BCR-ABL. Genes Dev. 2008;22(11):1411-1415. doi:10.1101/gad.1673908

21. Velasco-Hernández T, Vicente-Dueñas C, Sánchez-García I, Martin-Zanca D. p53 restoration kills primitive leukemia cells in vivo and increases survival of leukemic mice. Cell Cycle Georget Tex. 2013;12(1):122-132. doi:10.4161/cc.23031

22. Speck NA, Stacy T, Wang Q, et al. Core-binding factor: a central player in hematopoiesis and leukemia. Cancer Res. 1999;59(7 Suppl):1789s-1793s.

23. Roche-Lestienne $C$, Deluche L, Corm S, et al. RUNX1 DNA-binding mutations and RUNX1-PRDM16 cryptic fusions in $B C R-A B L+$ leukemias are frequently associated with secondary trisomy 21 and may contribute to clonal evolution and imatinib resistance. Blood. 2008;111(7):3735-3741. doi:10.1182/blood-2007-07-102533

24. Beer PA, Knapp DJHF, Miller PH, et al. Disruption of IKAROS activity in primitive chronic-phase CML cells mimics myeloid disease progression. Blood. 2015;125(3):504-515. doi:10.1182/blood-201406-581173

25. Georgopoulos K, Bigby M, Wang JH, et al. The Ikaros gene is required for the development of all lymphoid lineages. Cell. 1994;79(1):143-156.

26. Nakai H, Misawa S, Toguchida J, Yandell DW, Ishizaki K. Frequent p53 gene mutations in blast crisis of chronic myelogenous leukemia, especially in myeloid crisis harboring loss of a chromosome 17p. Cancer Res. 1992;52(23):6588-6593.

27. Sill H, Goldman JM, Cross NC. Homozygous deletions of the $\mathrm{p} 16$ tumor-suppressor gene are associated with lymphoid transformation of chronic myeloid leukemia. Blood. 1995;85(8):20132016.

28. Nakayama H, Ishimaru F, Avitahl N, et al. Decreases in Ikaros activity correlate with blast crisis in patients with chronic myelogenous leukemia. Cancer Res. 1999;59(16):3931-3934.

29. Togasaki E, Takeda J, Yoshida K, et al. Frequent somatic mutations in epigenetic regulators in newly diagnosed chronic myeloid leukemia. Blood Cancer J. 2017;7(4):e559. doi:10.1038/bcj.2017.36

30. Hernández-Boluda J-C, Cervantes F, Colomer D, et al. Genomic p16 abnormalities in the progression of chronic myeloid leukemia into blast crisis: a sequential study in 42 patients. Exp Hematol. 2003;31(3):204-210.

31. Kaelin WG. Gleevec: prototype or outlier? Sci STKE Signal Transduct Knowl Environ. 2004;2004(225):pe12. doi:10.1126/stke.2252004pe12

32. Yizhak K, Aguet F, Kim J, et al. RNA sequence analysis reveals macroscopic somatic clonal expansion across normal tissues. Science. 2019;364(6444). doi:10.1126/science.aaw0726

33. Jaiswal $\mathrm{S}$, Fontanillas $\mathrm{P}$, Flannick J, et al. Age-related clonal hematopoiesis associated with adverse outcomes. N Engl J Med. 2014;371(26):2488-2498. doi:10.1056/NEJMoa1408617 
34. Etzioni R, Urban N, Ramsey S, et al. Early detection: The case for early detection. Nat Rev Cancer. 2003;3(4):243-252. doi:10.1038/nrc1041

35. Stracci F. Cancer screenings, diagnostic technology evolution, and cancer control. Methods Mol Biol Clifton NJ. 2009;471:107-136. doi:10.1007/978-1-59745-416-2_6

36. Phallen J, Sausen M, Adleff V, et al. Direct detection of early-stage cancers using circulating tumor DNA. Sci Transl Med. 2017;9(403):eaan2415. doi:10.1126/scitransImed.aan2415 